\title{
Perfil de ácidos grasos libres y características sensoriales de quesos reggianito elaborados con diferentes fermentos
}

\author{
Por M.C. Perotti ${ }^{1 \star}$, S. Bernal, V. Wolf y C.A. Zalazar ${ }^{1}$ \\ Instituto de Lactología Industrial (INLAIN) \\ Facultad de Ingeniería Química. Universidad Nacional del Litoral/CONICET \\ $1^{\circ}$ de Mayo 3250, 3000M Santa Fe, Argentina \\ ( ${ }^{\star}$ autor para la correspondencia: cperotti@ fiq.unl.edu.ar)
}

\section{RESUMEN}

Perfil de ácidos grasos libres y características sensoriales de quesos Reggianito elaborados con diferentes fermentos.

El queso Reggianito es la variedad más importante dentro de los quesos duros fabricados en Argentina. Durante la maduración se producen innumerables reacciones bioquímicas sobre sus constituyentes, que conducen a definir las características sensoriales típicas de esta variedad de queso. Una de estas transformaciones involucra a la materia grasa originándose en una primera etapa ácidos grasos libres (AGL).

En el presente trabajo se evaluó el perfil de AGL desde $\mathrm{C}_{6: 0}$ hasta $\mathrm{C}_{18: 2}$ a diferentes tiempos de maduración, en quesos Reggianito elaborados con suero fermento natural y con cepas seleccionadas de Lactobacillus helveticus. La información obtenida de los perfiles cromatográficos fue analizada por Análisis por Componentes Principales (ACP) y Análisis Discriminante (AD). Los niveles de los AGL aumentaron significativamente $(p<0,05)$ durante la maduración, sin embargo no se encontraron diferencias entre los quesos producidos con distintos fermentos. El análisis sensorial indicó que todos los quesos fueron similares y presentaron una calidad sensorial satisfactoria.

Los resultados de este estudio además de contribuir al mejor conocimiento de la maduración del queso Reggianito, considerando la escasa información existente sobre la degradación de la materia grasa (lipólisis), realizan un significativo aporte para tratar de reemplazar los fermentos naturales por bacterias lácticas seleccionadas con todas las ventajas que ello significa para la estandarización y mejora de la calidad del producto.

PALABRAS-CLAVE: Características sensoriales - Fermentos seleccionados - Lipólisis - Queso Reggianito.

\section{SUMMARY}

Free fatty acid profile and sensory characteristics of Reggianito cheese produced with different starters.

Reggianito is the most important variety among the Argentinean hard cheeses. During its ripening period several biochemical reactions of proteins and fat are responsible for its final sensory characteristics. The fat transformations, in a first step, produce free fatty acids (FFA).

In the present work, the FFA profile from $\mathrm{C}_{6: 0}$ to $\mathrm{C}_{18: 2} \mathrm{du}$ ring ripening was evaluated. Reggianito cheeses were produced with a "wild" whey starter and with selected strains of Lactobacillus helveticus isolated from natural whey. Information from FFA profiles was processed by Principal Component Analysis (PCA) and Discriminant Analysis (DA). Despite the fact that FFA levels grew continuously during ripening, no differences were found among cheeses produced with different starters. Sensory analyses did not show differences among the different cheeses produced. All cheeses were of satisfactory sensory quality. Results from this study are important for an increased knowledge of the ripening process of Reggianito cheese, considering the scarce information currently existing on this subject. At the same time, the substitution of a natural whey starter for a selected strain starter appears to be a possible advantage in order to improve and to standardize the quality of the cheese.

KEY-WORDS: Lipolysis - Reggianito cheese - Selected starters - Sensory analysis.

\section{INTRODUCCIÓN}

La maduración de quesos es un proceso en el cual ocurren numerosas reacciones bioquímicas, sobre las proteínas, la materia grasa y la lactosa, responsables de importantes cambios en la textura y en las características sensoriales, que conducen a determinar la tipicidad de cada producto (Fox, 1993).

En ciertos quesos la degradación de la materia grasa, proceso conocido como lipólisis, es de gran relevancia en la formación de aroma y sabor, debido a la producción de ácidos grasos libres y de compuestos derivados de sus transformaciones. Este es el caso de los quesos adicionados de hongos, tales como Camembert, Cabrales, Roquefort y Gorgonzola, donde la lipolisis conduce a la formación de numerosos compuestos de gran influencia

\footnotetext{
${ }^{1}$ Investigadores del Consejo Nacional de Investigaciones Científicas y Técnicas (CONICET), Argentina.
} 
en los caracteres organolépticos (Collins et al., 2003; McSweeney y Sousa, 2000). Por otro lado, se tienen los quesos duros italianos tales como el Grana Padano y el Parmigiano Reggiano, en los cuales la hidrólisis que sufre la grasa es limitada. Sin embargo, y como consecuencia del prolongado período de maduración los agentes responsables de estas transformaciones pueden llegar a producir cambios significativos. Estos agentes son principalmente las bacterias del fermento natural de suero, las bacterias lácticas no pertenecientes al fermento (NSLAB) y la lipasa natural de la leche, debido a que estos quesos son elaborados con leche cruda (Battistoti y Corradini, 1993).

El queso Reggianito es la variedad más importante dentro de los quesos duros fabricados en Argentina, pudiendo considerarse descendiente de los productos italianos mencionados (Zalazar et al., 1999). Sin embargo, diferencias en la tecnología y en la calidad de la leche, le han otorgado a este producto características propias a tal punto que en la actualidad puede considerarse como un queso típico Argentino. En este producto, elaborado con leche pasteurizada, el fermento natural de suero es ampliamente usado, tal como ocurre en sus antecesores italianos (Battistoti y Corradini, 1993; Caboni et al., 1988). El empleo de estos fermentos naturales conlleva una serie de ventajas tales como la contribución importante al aroma y sabor, atribuido a la complejidad de su microflora, como así también una notable resistencia al ataque por fagos. Por otra parte, presentan algunas desventajas, tales como la variación en su composición microbiológica que incide en la carencia de uniformidad del producto final y la presencia de hongos y levaduras como contaminantes indeseables. Por esta razón, se han desarrollado numerosos estudios tendientes a preservar las ventajas de los fermentos naturales por un lado y minimizar sus efectos negativos por otro lado. En este sentido la inclusión de cepas seleccionadas en estos fermentos se ha empleado como una posible vía de solución al problema (Botazzi et al., 1999; Hynes et al., 2003; Reinheimer et al., 1995 y 1996).

En este trabajo se caracterizó el queso Reggianito elaborado con suero fermento natural, desde el punto de vista del perfil de ácidos grasos libres y del análisis sensorial. Además, se observaron los cambios que ocurren al reemplazar el fermento natural con cepas seleccionadas de Lactobacillus helveticus. El principal objetivo fue examinar las modificaciones en los principales AGL a lo largo de la maduración, para los diferentes fermentos empleados.

\section{MATERIALES Y MÉTODOS}

\section{Elaboración de quesos}

Quesos del tipo Reggianito fueron producidos mediante la tecnología estándar adaptada a escala piloto (Hynes et al., 2003). Se elaboraron quesos control empleando suero fermento natural (SFN) y tres tipos de quesos experimentales con suero fermento natural, libre de microorganismos, inoculado individualmente con tres cepas de bacterias lácticas seleccionadas (Lh 133, Lh 138 y Lh 209) (Reinheimer et al.,1995 y 1996).

Como materia prima se usaron $150 \mathrm{~L}$ de leche cruda entera (3,9 $\pm 0,2 \%$ de materia grasa), $\mathrm{pH}$ $6,60 \pm 0,05$ y $18^{\circ} \pm 1^{\circ} \mathrm{D}$ de acidez $\left(1^{\circ} \mathrm{D}=100 \mathrm{mg}\right.$ de ácido láctico $\mathrm{L}^{-1}$ ), suministrada por un establecimiento lácteo de la zona de Santa Fe (Argentina). El contenido de materia grasa de la leche se estandarizó a $2,5 \%$ y se pasteurizó en sistema discontinuo a $65{ }^{\circ} \mathrm{C}$ durante $20 \mathrm{~min}$. La leche pasteurizada se repartió en dos tinas queseras a razón de $75 \mathrm{~L}$ en cada una. Después de enfriar a $33^{\circ} \mathrm{C}$, se adicionó cloruro de calcio hasta una concentración final de $0,02 \%(p / v)$. Se agregó el fermento correspondiente (aprox. $4 \% \mathrm{v} / \mathrm{v}$ del volumen de leche) y después de $10 \mathrm{~min}$. de agitación mecánica se adicionó coagulante líquido de bovino adulto 230 IMCU $\mathrm{mL}^{-1}$ (Naturen, Chr. Hansen de Argentina S.A.) $\left(0,29 \mathrm{~mL} \mathrm{~L}^{-1}\right.$ de leche $)$. Luego de $18-20 \mathrm{~min}$. se procedió al corte de la cuajada en forma manual, la mezcla se sometió a calentamiento bajo constante agitación hasta $44-45^{\circ} \mathrm{C}\left(0,5^{\circ} \mathrm{C} \mathrm{min}^{-1}\right)$ para reducir la humedad de los granos de cuajada, se calentó más rápidamente $\left(1^{\circ} \mathrm{C} \mathrm{min}^{-1}\right)$ hasta $52{ }^{\circ} \mathrm{C}$ y alcanzada dicha temperatura se interrumpió la agitación. Las cuajadas se separaron del suero y se pasaron a moldes cilíndricos donde se prensaron durante $24 \mathrm{~h}$. Los quesos se desmoldaron y se sumergieron en salmuera $(\mathrm{NaCl} 20 \% \mathrm{p} / \mathrm{v}, \mathrm{pH} 5,4) \mathrm{du}-$ rante 6 días a $12{ }^{\circ} \mathrm{C}$. La maduración se llevó a cabo en cámara a $12{ }^{\circ} \mathrm{C}$ y $80 \%$ de humedad relativa por 180 días.

Para cada tipo de queso (control y tres experimentales) se fabricaron tres réplicas, de manera de minimizar la influencia de variables incontroladas tales como tipo de leche, factores ambientales, etc., con lo que se tuvo un total de 12 unidades experimentales (quesos) que se distribuyeron en 6 días de elaboración (dos quesos por día, de aprox. $6 \mathrm{~kg}$ cada uno). La distribución de los quesos en los días de elaboración se realizó con un modelo completamente aleatorio.

Se tomaron muestras de la cuajada inmediatamente después de la elaboración y de los quesos a los 90 y 180 días de maduración de acuerdo al método estándar IDF (FIL-IDF, 50C:1995), y se conservaron $\mathrm{a}-18^{\circ} \mathrm{C}$ hasta la realización del análisis.

\section{Composición global}

Se determinó la composición global de los quesos control y experimentales a los 180 días de maduración. Se realizaron los análisis de extracto se$\mathrm{co}$, materia grasa y proteínas totales, con métodos normalizados (FIL-IDF 4A:1982; Bradley, 1993; FILIDF 20B:1993, respectivamente). 


\section{Recuentos microbiológicos}

La población total de bacterias lácticas termófilas presentes en las cuajadas y en los quesos a los 90 y 180 días de maduración, fue determinada sembrando diluciones de las muestras en placas conteniendo agar-leche descremada y contando las colonias luego de 48 horas de incubación a $37^{\circ} \mathrm{C}$, según los estándares de APHA (Frank et al., 1993).

\section{Evaluación de la lipolisis}

Los ácidos grasos libres (AGL) desde $\mathrm{C}_{6: 0}$ hasta $\mathrm{C}_{18: 2}$ presentes en los quesos control y experimentales, se extrajeron de la muestra acidificada con nhexano en caliente y se cuantificaron como ésteres etílicos por cromatografía de gases. Para el análisis de los ácidos grasos se empleó una columna capilar de sílice fundida $(30 \mathrm{~m} \times 0,25 \mathrm{~mm} \times 0,25 \mu \mathrm{m})$ empacada con polietilenglicol, usando un cromatógrafo de gases Perkin Elmer serie 9000 equipado con un detector de ionización de llama (FID), un inyector split-splitless, nitrógeno como gas carrier y software Turbocrom v4.0. La temperatura inicial del horno fue de $50{ }^{\circ} \mathrm{C}$ por $4 \mathrm{~min}$, se incrementó hasta $150{ }^{\circ} \mathrm{C}$ a razón de $10^{\circ} \mathrm{C} \mathrm{min}^{-1}$ manteniéndose a esta temperatura por $3 \mathrm{~min}$, se volvió a aumentar hasta $230^{\circ} \mathrm{C}$ a razón de $10^{\circ} \mathrm{C} \min ^{-1}$ y se mantuvo a esta temperatura final por $5 \mathrm{~min}$. La temperatura del inyector fue de $220^{\circ} \mathrm{C}$ en modo split (1/52) y el detector se termostatizó $275^{\circ} \mathrm{C}$ (Perotti et al., 2005). La concentración en $\mathrm{mg} \mathrm{kg}^{-1}$ de queso para los 9 AGL se calculó con el método del estándar interno $\left(\mathrm{C}_{7: 0}\right.$ y $\left.\mathrm{C}_{17: 0}\right)$ empleando curvas de calibrado para cada ácido.

\section{Evaluación sensorial}

Se determinaron las características sensoriales de los quesos control y experimentales al final de la maduración. El análisis se realizó por duplicado con un panel de 8 integrantes entrenados para la degustación de quesos duros, empleando un análisis descriptivo cuantitativo. Cada atributo se cuantificó utilizando una escala no estructurada de $10 \mathrm{~cm}$ anclada en los extremos. Se analizaron los siguientes atributos: aroma genuino, color, textura visual, textura oral, fracturabilidad, corte granular, flavor genuino, gusto salado y flavor residual.

\section{Procesamiento estadístico de los resultados}

El grado de lipólisis global expresado como la suma de las concentraciones de los 9 AGL cuantificados, se analizó con análisis de varianza usando el tiempo de maduración y los fermentos empleados como factores principales.

Los perfiles de lipólisis se analizaron más profundamente empleando técnicas multivariadas (análisis por componentes principales y análisis discriminante), con el propósito de estudiar las diferencias entre los quesos y las variaciones con el tiempo de maduración (software SPSS v. 10.0, SPSS Inc., Chicago, USA).

Los resultados obtenidos del análisis sensorial se procesaron aplicando análisis de variancia a cada atributo, con el objetivo de detectar diferencias entre los distintos fermentos.

Además, se investigó la posible existencia de relaciones entre los ácidos grasos libres volátiles $\left(\mathrm{C}_{6: 0}\right.$ y $\mathrm{C}_{8: 0}$ ) y los atributos de aroma y flavor genuino, mediante la aplicación de técnicas de regresión simple y múltiple.

\section{RESULTADOS Y DISCUSIÓN}

\section{Composición global y microbiología}

Los valores promedio y desviación estándar para extracto seco, materia grasa y proteína total de los quesos al final de la maduración, se presentan en la Tabla 1. Estos parámetros fisicoquímicos se encontraron dentro de los valores establecidos por la Legislación Argentina para la variedad Reggianito (Código Alimentario Argentino, 2007). Los resultados obtenidos para cada uno de los parámetros se analizaron con análisis de varianza. No se encontraron diferencias significativas en la composición global de los quesos elaborados con fermento natural de suero (quesos control) y con fermentos seleccionados (quesos experimentales).

El recuento de bacterias lácticas termófilas en las cuajadas antes del moldeo arrojó valores de aproximadamente $10^{7}$ UFC $\mathrm{g}^{-1}$ para los quesos control y para aquellos en los que se emplearon las cepas Lh 133 y Lh 209. En cambio, las cuajadas con Lh 138, mostraron recuentos de $10^{8} \mathrm{UFC}^{-1}$. Todos los quesos alcanzaron recuentos de $10^{8}$ UFC $\mathrm{g}^{-1}$ a las 24 horas, para luego decrecer uno o

Tabla 1

Composición global de quesos Reggianito control y experimentales a los 180 días de maduración

\begin{tabular}{lcccc}
\hline & \multirow{2}{*}{ Quesos control } & \multicolumn{3}{c}{ Quesos experimentales } \\
\cline { 3 - 4 } & & Lh 133 & Lh 138 & Lh 209 \\
\hline Extracto seco (\%) & $62.97 \pm 0.58^{\mathrm{a}}$ & $64.55 \pm 2.53^{\mathrm{a}}$ & $64.44 \pm 2.29^{\mathrm{a}}$ & $63.23 \pm 3.63^{\mathrm{a}}$ \\
Materia grasa (\%) & $23.58 \pm 1.09^{\mathrm{a}}$ & $26.82 \pm 2.60^{\mathrm{a}}$ & $25.00 \pm 2.77^{\mathrm{a}}$ & $23.29 \pm 1.26^{\mathrm{a}}$ \\
Proteína total (\%) & $28.94 \pm 1.03^{\mathrm{a}}$ & $28.73 \pm 1.54^{\mathrm{a}}$ & $29.61 \pm 0.26^{\mathrm{a}}$ & $29.69 \pm 1.75^{\mathrm{a}}$ \\
\hline
\end{tabular}

${ }^{a}$ Los promedios en una misma fila con igual letra superíndice, no son significativamente diferentes $(p>0,05)$. 
dos órdenes durante la maduración. Los quesos experimentales con la cepa Lh 209 mostraron al final de la maduración los recuentos más bajos, alrededor de $10^{6}$ UFC g $^{-1}$.

\section{Evaluación de la lipólisis. Análisis multivariado}

En el estudio de la lipólisis se analizaron 12 quesos (tres réplicas de los cuatro tipos) a tres tiempos de maduración (0, 90 y 180 días), obteniéndose un total de 36 perfiles cromatográficos de los que se calcularon las concentraciones de $9 \mathrm{AGL}$. Estos resultados se analizaron desde un aspecto univariado y también multivariado.

En la Figura 1 puede apreciarse la evolución de los AGL totales, calculado como la suma los 9 AGL cuantificados, en los distintos tipos de quesos durante la maduración. Resulta evidente el aumento de la concentración de AGL totales a medida que transcurre este proceso.
En la Tabla 2 se presentan los resultados para las concentraciones de los AGL ( $\mathrm{mg} \mathrm{kg}^{-1}$ de queso) desde $\mathrm{C}_{6: 0}$ hasta $\mathrm{C}_{18: 2}$ a los 180 días de maduración, para los quesos elaborados en el presente trabajo (escala piloto). Los AGL más abundantes encontrados en las muestras, independiente del fermento empleado en los quesos, fueron los ácidos oleico $\left(C_{18: 1}\right)$, palmítico $\left(C_{16: 0}\right)$, esteárico $\left(C_{18: 0}\right)$ y mirístico $\left(C_{14: 0}\right)$, que representaron en conjunto el $86 \%$ del total. Para el queso Reggianito elaborado con Lh 138 se obtuvieron concentraciones de AGL significativamente superiores ( $p<0,05$ ) en comparación con los otros quesos. En esta tabla también se muestra el perfil de AGL de queso Reggianito elaborado a escala industrial (Sihufe et al., 2007). Si se comparan los perfiles obtenidos para los quesos a escala piloto con los obtenidos en la industria, se observa que son comparables, hecho que permitiría extrapolar a escala industrial los resultados obtenidos a escala piloto.

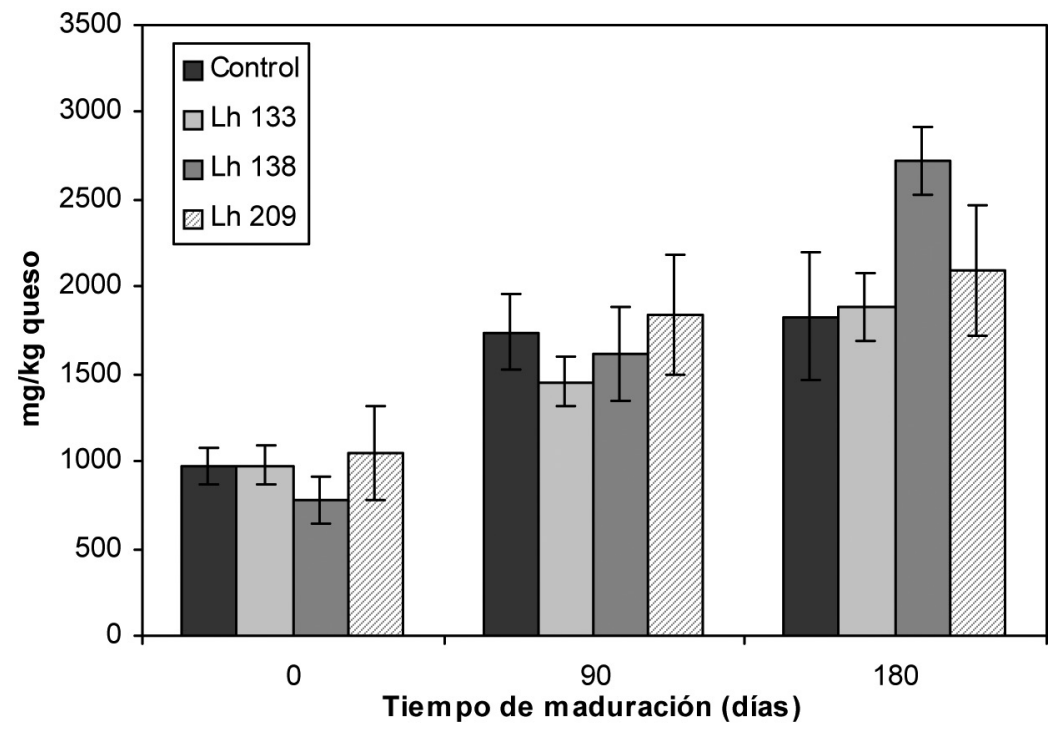

Figura 1

Evolución de los AGL totales durante la maduración en los distintos quesos elaborados a escala planta piloto

Tabla 2

Perfil de lipólisis de quesos Reggianito elaborados a escala piloto y a escala industrial, con 180 días de maduración

\begin{tabular}{|c|c|c|c|c|c|c|c|c|c|}
\hline $\begin{array}{l}\text { Ácidos Grasos } \\
\text { Libres } \\
\text { (mg kg-1 queso) }\end{array}$ & $\mathrm{C}_{6: 0}$ & $\mathrm{C}_{8: 0}$ & $C_{10: 0}$ & $C_{12: 0}$ & $C_{14: 0}$ & $C_{16: 0}$ & $C_{18: 0}$ & $C_{18: 1}$ & $C_{18: 2}$ \\
\hline \& Control & $30,3 \pm 6,6^{a}$ & $26,7 \pm 6,1^{\mathrm{a}}$ & $56,5 \pm 13,3^{a}$ & $71,1 \pm 11,2^{\mathrm{a}}$ & $164,8 \pm 19,5^{\mathrm{a}}$ & $565,9 \pm 114,2^{a}$ & $240,7 \pm 55,8^{\mathrm{a}}$ & $606,0 \pm 107,7^{a}$ & $56,4 \pm 7,8^{a}$ \\
\hline 힝 흥 $\operatorname{Lh} 133$ & $40,3 \pm 8,9^{a}$ & $28,6 \pm 5,6^{\mathrm{a}}$ & $63,6 \pm 12,5^{\mathrm{a}}$ & $70,5 \pm 16,7^{\mathrm{a}}$ & $184,5 \pm 37,6^{a}$ & $620,6 \pm 54,4^{\mathrm{a}}$ & $259,3 \pm 14,5^{\mathrm{a}}$ & $613,8 \pm 62,2^{\mathrm{a}}$ & $77,5 \pm 11,3^{\mathrm{a}, \mathrm{b}}$ \\
\hline 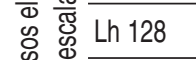 & $64,5 \pm 16,4^{b}$ & $47,9 \pm 11,1^{b}$ & $90,9 \pm 11,5^{b}$ & $101,0 \pm 12,1^{b}$ & $317,3 \pm 95,2^{b}$ & $841,6 \pm 27,5^{b}$ & $336,1 \pm 43,1^{b}$ & $839,3 \pm 115,2^{a, b}$ & $106,4 \pm 41,6^{b}$ \\
\hline 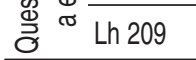 & $39,8 \pm 14,2^{a}$ & $29,5 \pm 8,6^{a}$ & $61,3 \pm 13,0^{a}$ & $64,2 \pm 13,0^{a}$ & $229,5 \pm 53,5^{a, b}$ & $656,2 \pm 115,7^{\mathrm{a}}$ & $232,9 \pm 31,4^{\mathrm{a}}$ & $670,0 \pm 73,7^{\mathrm{a}}$ & $92,5 \pm 23,3^{a, b}$ \\
\hline $\begin{array}{l}\text { Quesos } \\
\text { elaborados } \\
\text { a escala } \\
\text { industrial }\end{array}$ & $48,7 \pm 9,9$ & $34,6 \pm 1,8$ & $68,8 \pm 2,1$ & $78,1 \pm 7,3$ & $300,3 \pm 39,5$ & $727,1 \pm 65,9$ & $258,8 \pm 18,9$ & $776,3 \pm 90,8$ & $106,2 \pm 9,8$ \\
\hline
\end{tabular}

\footnotetext{
${ }^{a, b}$ Los promedios en una misma columna con diferente letra superíndice, son significativamente diferentes $(p<0,05)$.
} 
En la Tabla 3 se observa el porcentaje de cada AGL respecto al valor de AGL totales. Estos valores se compararon con el perfil de ácidos grasos de la grasa láctea según MacGibbon y Taylor (2006), pudiéndose notar que son similares. Con este análisis se concluye que la hidrólisis enzimática de la materia grasa que ocurre durante el proceso de maduración no es selectiva, ya que no se utilizaron lipasas exógenas que podrían efectuar una liberación preferencial de ácidos grasos a partir de la grasa de la leche.

Para profundizar el análisis de los resultados se construyó una matriz de datos multivariados con los 36 perfiles de lipólisis, compuesta de 36 filas (muestras) y 9 variables (ácidos grasos libres cuantificados), que se sometió al análisis por componentes principales (ACP). El ACP es un método estadístico multivariado cuya finalidad es simplificar las múltiples relaciones que puedan existir entre las variables observadas encontrando nuevas direcciones (componentes o factores) con mínima pérdida de la información facilitando la interpretación de los datos. Para la extracción de los componentes se empleó la matriz correlación, ya que los 9 ácidos grasos libres tenían valores medios y varianzas con órdenes de magnitud muy diferentes. Con este procedimiento se consiguió la estandarización de las variables a media cero y varianza unitaria. Los contrastes de esfericidad de Barlett y la medida de adecuación muestral de KaiserMeyer-Olkin (KMO) se calculan previamente a la extracción de los componentes, para analizar la pertinencia de la aplicación del ACP. Los valores obtenidos para estos dos parámetros fueron grandes (642 para el estadístico chi cuadrado del test de Barlett y 0,81 para KMO), lo que confirmó que las variables estaban altamente correlacionadas pudiéndose explicar así la variabilidad de la matriz con un número menor de variables o componentes (Hair et al. 1999, Pérez López, 2004). Se seleccionaron tres componentes principales que explicaron el $96,1 \%$ de la variabilidad total del sistema, representando $\mathrm{CP}_{1}$ el $82,9 \%, \mathrm{CP}_{2}$ el $10,2 \%$ y $\mathrm{CP}_{3}$ el $3,0 \%$, respectivamente. Los 9 ácidos grasos fueron considerados en el análisis debido a que sus comunalidades (porcentaje de varianza de cada variable explicada por los tres primeros componentes) fueron altas (superiores a 0,92). Todos los ácidos tuvieron cargas factoriales elevadas y positivas en $\mathrm{CP}_{1}$ (caproico, 0,96; caprílico, 0,93; cáprico,
0,91; láurico, 0,89; mirístico, 0,86; palmítico, 0,96; esteárico, 0,93; oleico, 0,95 y linoleico, 0.80), no sucediendo lo mismo para $\mathrm{CP}_{2}$ ya que solamente se encontraron valores intermedios para cuatro de los nueve ácidos, positivos para dos de ellos y negativos para los otros dos (linoleico, 0,49; mirístico, 0,46 , láurico, $-0,43$ y cáprico $-0,38)$. Los restantes $A G L$ tuvieron cargas factoriales pequeñas en este componente. En relación a $\mathrm{CP}_{3}$ se encontraron valores ligeramente bajos para dos ácidos (linoleico, 0,27 y esteárico, $-0,30$ ), y los restantes presentaron cargas factoriales inferiores a estos valores.

Posteriormente, se realizó la rotación de los componentes con el método VARIMAX de manera de maximizar las cargas factoriales de cada una de las variables en un único componente. La rotación afecta solamente la distribución de las proporciones de la varianza total explicada por cada componente, pero se mantiene la proporción acumulada de varianza total explicada por los tres CPs. Después del procedimiento de rotación, las cargas factoriales de todos los ácidos en los tres componentes fueron positivas. En $\mathrm{CP}_{1}(43,1 \%$ de la varianza) contribuyeron de manera importante los ácidos de cadena corta (caproico, 0.69; caprílico, 0,88; cápri$\mathrm{co}, 0,91$ y laúrico, 0,90$)$, en $\mathrm{CP}_{2}(33,6 \%)$ mirístico $(0,84)$ y linoleico $(0,92)$. El ácido esteárico fue importante únicamente en $\mathrm{CP}_{3}(19,4 \%)$ con un valor de 0.69. Los ácidos de cadena larga que presentaron los mayores valores de concentración en la lipólisis, participaron en forma compartida en $\mathrm{CP}_{2} \mathrm{y}$ $\mathrm{CP}_{3}$ para palmítico $(0,62$ y 0,61 , respectivamente) y en los tres CPs para oleico $(0,52,0,58$ y 0,57 , respectivamente).

Se calcularon los "scores" o puntuaciones de las muestras de quesos en los $\mathrm{CP}_{1}, \mathrm{CP}_{2}$ y $\mathrm{CP}_{3}$ rotados, se graficaron las tres combinaciones posibles de estos CPs en dos dimensiones y se analizó cual de ellas mostraba una mejor separación entre los grupos de muestras. El mapa factorial que mejor representó esta separación fue la combinación $\mathrm{CP}_{1}$ versus $\mathrm{CP}_{2}$ (Figura 2), donde las muestras se identificaron por tiempo y por tipo de queso. Se observaron diferencias por tiempo de maduración de acuerdo a los dos ejes. El origen de coordenadas representa un perfil de lipólisis promedio. Las cuajadas se agruparon en el cuadrante negativo tanto para $\mathrm{CP}_{1}$ como para $\mathrm{CP}_{2}$, las muestras de maduración intermedia se localizaron cer-

Tabla 3

Porcentajes de AGL con respecto a los AGL totales para quesos Reggianito y porcentajes de AG en la grasa de leche

\begin{tabular}{|c|c|c|c|c|c|c|c|c|c|}
\hline Ácidos Grasos Libres & $\mathrm{C}_{6: 0}$ & $C_{8: 0}$ & $C_{10: 0}$ & $C_{12: 0}$ & $\mathrm{C}_{14: 0}$ & $C_{16: 0}$ & $C_{18: 0}$ & $\mathrm{C}_{18: 1}$ & $\mathrm{C}_{18: 2}$ \\
\hline $\begin{array}{l}\text { Quesos Reggianito } \\
\text { (promedio para los } \\
\text { cuatro tipos a } 180 \text { días) }\end{array}$ & 2,0 & 1,5 & 3,1 & 3,5 & 10,3 & 31,4 & 12,7 & 31,7 & 3,8 \\
\hline Grasa de leche (1) & 2,5 & 1,5 & 3,2 & 3,6 & 11,1 & 27,9 & 12,2 & 21,1 & \\
\hline
\end{tabular}

(1) MacGibbon y Taylor, 2006. 


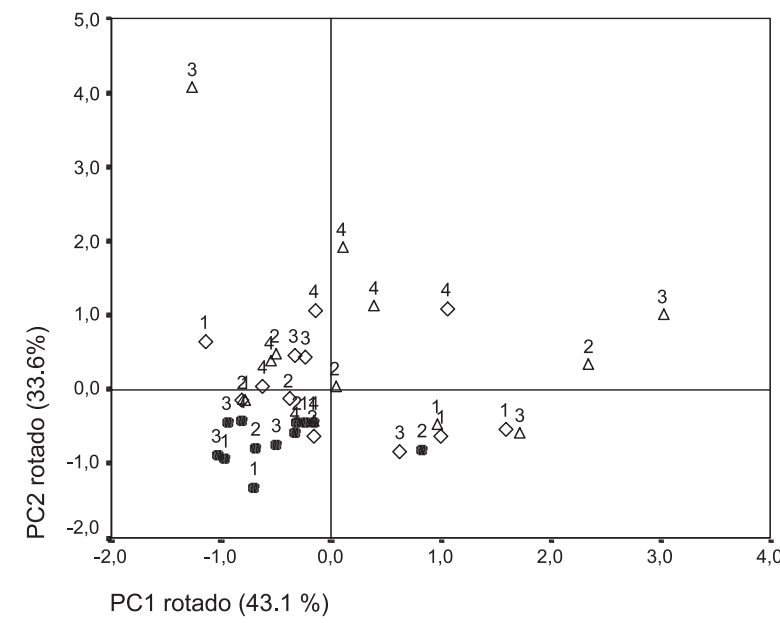

- Cuajadas; $\diamond$ quesos de 90 días y $\triangle$ quesos de 180 días de maduración. Quesos control (1), experimentales con Lh 133 (2), con Lh 138 (3) y con Lh 209 (4)

\section{Figura 2}

Gráfica de "scores" de las muestras de quesos definidas por los dos primeros componentes principales rotados $\mathrm{CP}_{1}$ y $\mathrm{CP}_{2}$, obtenida en el análisis por componentes principales de los perfiles de ácidos grasos libres (AGL) de quesos Reggianito (escala piloto) $(n=36)$

ca del origen de coordenadas y las de final de maduración se ubicaron en su mayoría en el cuadrante positivo con perfiles superiores al promedio. De este análisis se puede inferir que los perfiles de lipolisis aumentaron con el tiempo en el sentido positivo de $\mathrm{CP}_{3}$. Respecto a la diferenciación de las muestras por tipo de queso, se aprecia una separación en los cuatro grupos predefinidos aunque más débil que con el tiempo, lo que indicaría perfiles de lipólisis algo similares entre los distintos tipos de quesos.

A fin de validar la conclusión anterior basada en la observación visual de la gráfica de "scores", se aplicó el análisis discriminante lineal (DA) usando como variables predictoras los tres componentes principales seleccionados y como factor de clasificación el tiempo y el tipo de fermento. El análisis discriminante es una técnica estadística que se usa para clasificar individuos u objetos en grupos únicos y mutuamente excluyentes sobre la base de un set de variables independientes, donde se obtiene una combinación lineal de las mismas que discrimina entre los grupos definidos a priori de tal forma que se minimicen clasificaciones erróneas.

Con respecto al factor tiempo, se encontró una función discriminante significativa $(p<0,05)$ que permitió clasificar correctamente el $80,6 \%$ de las muestras en los 3 grupos predefinidos, el 100\% de las cuajadas, el $83 \%$ de las muestras con 90 días y el $58 \%$ de las de 180 días de maduración. La situación no fue la misma en relación al tipo de fermento utilizado en las muestras de quesos, ya que no se encontró una función discriminante significativa. Sin embargo, a partir de la gráfica de $\mathrm{PC}_{1}$ y $\mathrm{PC}_{2}$ scores es posible estimar visualmente algunas diferencias. Los quesos con 180 días de maduración y producidos con cepas de Lh 138 (ver figura 2), se localizaron más alejadas del origen de coordenadas y mostraron los mayores valores de scores para estos componentes. Esta situación podría ser interpretada como una actividad lipolítica mayor de Lactobacillus helveticus 138 en relación con las otras cepas estudiadas.

\section{Análisis sensorial}

Los valores promedio y desviaciones estándar de los diferentes atributos sensoriales analizados al final de la maduración, se presentan en la Tabla 4.

De los atributos analizados, solamente para flavor residual se encontró diferencia significativa $(p<0,05)$ entre los distintos fermentos empleados. Los valores más intensos se hallaron para los quesos elaborados con los fermentos Lh 133 y Lh 138, y menor puntuación para Lh 209. Sin embargo los panelistas no consideraron que estas diferencias

Tabla 4

Análisis sensorial de quesos Reggianito elaborados a escala piloto, a los 180 días de maduración

\begin{tabular}{|c|c|c|c|c|c|}
\hline \multirow{2}{*}{ Atributo } & \multirow{2}{*}{ Control } & \multicolumn{3}{|c|}{ Experimentales } & \multirow{2}{*}{$\begin{array}{c}\text { Nivel de } \\
\text { significancia }\end{array}$} \\
\hline & & Lh 133 & Lh 138 & Lh 209 & \\
\hline Aroma genuino & $5,0 \pm 0,5$ & $5,0 \pm 0,2$ & $4,6 \pm 0,5$ & $5,4 \pm 0,2$ & NS \\
\hline Color & $5,4 \pm 0,2$ & $5,5 \pm 0,3$ & $4,8 \pm 0,9$ & $5,1 \pm 0,5$ & NS \\
\hline Textura visual & $7,9 \pm 0,2$ & $7,9 \pm 0,1$ & $7,9 \pm 0,4$ & $7,1 \pm 0,2$ & $\mathrm{NS}^{*}$ \\
\hline Corte granular & $7,3 \pm 0,4$ & $7,5 \pm 0,2$ & $7,0 \pm 0,7$ & $7,1 \pm 0,7$ & NS \\
\hline Fracturabilidad & $8,2 \pm 0,3$ & $8,1 \pm 0,2$ & $7,8 \pm 0,7$ & $8,3 \pm 0,2$ & NS \\
\hline Textura oral & $7,5 \pm 0,2$ & $7,2 \pm 2,0$ & $6,7 \pm 0,4$ & $7,3 \pm 0,4$ & NS \\
\hline Flavor genuino & $8,1 \pm 0,4$ & $8,0 \pm 0,3$ & $7,7 \pm 0,4$ & $7,9 \pm 0,4$ & NS \\
\hline Fravor residual & $2,3 \pm 0,4^{a}$ & $4,2 \pm 0,3^{b}$ & $3,6 \pm 0,5^{b}$ & $1,0 \pm 0,3^{c}$ & $\star *$ \\
\hline Gusto salado & $5,9 \pm 0,5$ & $5,9 \pm 0,2$ & $5,9 \pm 0,1$ & $6,0 \pm 0,2$ & NS \\
\hline
\end{tabular}

NS: no significativamente diferentes, nivel de confianza 0,05 . NS *: no significativamente diferentes, nivel de confianza $0,01 .{ }^{*}$ : significativamente diferentes, $p<0,05$.

Las escalas se establecieron de la siguiente forma: aroma genuino ( 1 = débil, 9 = muy intenso), color $(1=$ débil, $9=$ muy intenso), textura visual: grietas mecánicas y ojos $(1=$ mucho, $9=$ nada), corte granular $(1=$ no granular, $9=$ muy granular $)$, fracturabilidad $(1=$ nada, $9=$ mucho), textura oral ( 1 = áspero, arenoso, pastoso, 9 = no áspero, no arenoso, no pastoso), flavor genuino: sabroso, salado y ligeramente picante $(1=$ extremadamente débil, $9=$ normalmente intenso), gusto salado $(1=$ débil, $9=$ intenso), flavor residual $(1=$ ausente, $9=$ mucho). 
justificaban una diferenciación neta de estos quesos con respecto al resto.

De este análisis se puede inferir que los quesos control y experimentales se pueden considerar similares. Los mayores valores encontrados para los AGL de los quesos con Lh 138, no se tradujeron en diferencias apreciables desde el punto de vista sensorial.

\section{Análisis de relaciones entre lipólisis y caracteres sensoriales}

De acuerdo a lo demostrado por diferentes autores algunos ácidos grasos libres, especialmente los de cadena corta aunque están presentes en baja concentración, contribuyen directamente al flavor de quesos muy madurados, o bien influyen positivamente en el sabor de fondo de ciertos quesos como el Cheddar (Fox et al., 1993; Collins et al., 2003). Por esta razón, se trató de encontrar la existencia de una relación entre los $A G L$ de cadena corta $\left(C_{6: 0}\right.$ y $\mathrm{C}_{8: 0}$ ) y el atributo de aroma y flavor genuino. Para ello se aplicaron métodos de regresión lineal simple y múltiple, considerando estos dos atributos como variables dependientes y los ácidos como variables independientes. En ninguno de los casos se obtuvo un modelo de regresión lineal que permitiera explicar la variabilidad en el aroma y flavor, ya que los coeficientes de correlación obtenidos fueron muy bajos indicando una falta de asociación entre las variables. Esto se justifica por el hecho que la contribución de los ácidos al aroma es difícil de definir, debido a distintos factores como las características de la pasta, el pH, el tenor de calcio, el contenido de humedad y porcentaje de grasa, el grado de maduración, la repartición de los ácidos entre la fase grasa y acuosa, la asociación con las proteínas y péptidos, entre otros (McSweeney y Sousa, 2000; Collins et al, 2003, Deeth y Fitz-Gerald, 2006).

Por otro lado, los quesos que se analizaron en este trabajo (control y experimentales), presentan un grado de lipólisis discreto, muy lejano al obtenido para productos en donde la acción enzimática es considerada muy importante y para los cuales se han informado buenas correlaciones entre los AGL y caracteres sensoriales (Collins et al., 2003; Caboni et al., 1988).

\section{CONCLUSIONES}

En este trabajo se presentan perfiles de ácidos grasos libres y características sensoriales para quesos Reggianito elaborados a escala piloto, con suero fermento natural y con fermentos seleccionados de Lactobacillus helveticus. Se compararon estos resultados con los obtenidos para quesos a escala industrial, no observándose diferencias apreciables entre las concentraciones de AGL.

Los resultados obtenidos resultaron útiles para comprender mejor el proceso de maduración de este queso duro típico argentino, dada la escasa información disponible en este sentido.
Se pudo establecer una escasa diferencia desde el punto de vista de la transformación primaria de la materia grasa entre los productos elaborados con suero fermento natural y con fermentos seleccionados aislados del propio suero. Estos resultados permiten que se pueda considerar con muy buenas perspectivas el reemplazo de los fermentos naturales por los seleccionados con todas las ventajas que ello implica sobre la calidad del queso pero conservando las características sensoriales típicas del producto. Esta posibilidad se ve más reforzada si se consideran los resultados que para el mismo reemplazo se obtuvieron del estudio de la proteolisis (Hynes et al., 2003).

Los mayores valores observados para los quesos elaborados con el fermento Lh 138 , no se tradujeron sin embargo, en diferencias desde el punto de vista sensorial, lo que indicaría que los AGL no son únicamente los compuestos que más influyen sobre características tales como aroma y sabor.

\section{AGRADECIMIENTOS}

Los autores agradecen a la Lic. N. Sabbag por su colaboración en el análisis sensorial de los quesos.

\section{BIBLIOGRAFÍA}

Battistotti B. Corradini C. 1993. Italian cheese. En P. Fox (Ed.). Cheese: Chemistry, Physics and Microbiology. Vol. 2 (pag. 221-243). Chapman \& Hall, London (1993).

Bottazzi V, Parisi M. Cocconcelli P. 1999. Colture di batteri lattici per la produzione di fromaggio grana e tipizzazione molecolari dei ceppi. Scienza e Técnica Lattiero-Casearia 50, 179-194.

Bradley RL, Arnold E, Barbano DM, Semerad RG, Smith DE. Vines, B.K. 1993. Chemical and physical methods. Standard methods for the examination of dairy products, pag. 433-453. American Public Health Association, Washington DC, Ed. R. Marshall.

Caboni MF, Zannoni M. Lercker G. 1988. Lipolisi del grasso del Parmigiano-Reggiano en Atti giornata studio composizione e caractteristiche Parmigiano-Reggiano, pag. 113-121, Cons. Form. Parmigiano-Reggiano (Ed.). Regio Emilia.

Collins YF, McSweeney PLH. Wilkinson M.G. 2003. Lipolysis and free fatty acid catabolism in cheese: a review of current knowledge. International Dairy Journal 13 (11) 841-866.

Deeth H. Fitz-Gerald C. 2006. Enzymes and hydrolityc rancidity. En P. Fox y P. McSweeney (Ed.). Advanced Dairy Chemistry. Vol. 2 Lipids (pag. 481-530). Springer Science, New York.

FIL-IDF 50C: Lait et produits Laitiers. Guide de L'echantillonnage. Brussels, Belgium (1995).

FIL-IDF 4A: Formaggio e formaggio fuso. Determinazione della matria secca. Metodo di riferimento. Brussels, Belgium (1982).

FIL-IDF 20B: Latte. Determinazione del tenore in azoto. Metodo di riferimento. Brussels, Belgium (1993).

Fox PF. Law J, McSweeney P. Wallace J. 1993. Biochemistry of cheese ripening. En P. F. Fox (Ed.). Cheese: 
Chemistry, Physics and Microbiology. Vol. 1 (pag. 389). General Aspects. Chapman \& Hall, London.

Frank J, Christen G. Bullerman L. 1993. Test for groups of microorganism. In R. Marshall (Ed.). Standard methods for the examination of dairy products. American Public Health Association, Washington D.C.

Hair J, Anderson R, Tatham R. Black W. 1999. Análisis Multivariado, Otero (Ed.), Prentice Hall, Madrid.

Hynes E, Bergamini C, Suárez V. Zalazar C. 2003. Proteolysis in Reggianito Argentino cheeses manufactured with natural whey cultures and selected strains of Lactobacillus helveticus. Journal of Dairy Science 86, 38313840 .

MacGibbon A. Taylor M. 2006. Composition and structure of bovine milk lipids. En P. F. Fox and P. McSweeney (Ed.). Advanced Dairy Chemistry. Vol. 2 Lipids (pag. 32). Springer Science, New York.

McSweeney P. Sousa M. 2000. Biochemical pathways for the production of flavour compounds in cheese during ripening: a review. Le Lait 80, 293-324.

CAA, Código Alimentario Argentino (2007). http:// www.anamt.gov.ar/codigoa/caa1.htm.

Pérez López C. 2004. Técnicas Estadísticas con SPSS ${ }^{\circledR}$, Pearson (Ed.), Prentice Hall, Madrid.
Perotti MC, Bernal SM, Meinardi CA. Zalazar CA. 2005. Free fatty acid profiles of Reggianito Argentino cheese produced with different starters. International Dairy Journal 15, $1150-1155$.

Reinheimer J, Quiberoni A, Tailliez P, Binetti A. Suárez V. 1996. The lactic acid microflora of natural whey starters used in Argentina for hard cheese production. International Dairy Journal 6, $869-879$.

Reinheimer J, Suárez V, Bailo N. Zalazar C. 1995. Microbiological and Technological characteristics of natural whey cultures for Argentinian hard cheese production. Journal of Food Protection 58 (7) 796 - 799.

Sihufe G, Zorrilla S, Mercanti D, Perotti M.C, Zalazar, CA. Rubiolo A. 2007. The influence of ripening temperature and sampling site on the lipolysis in Reggianito Argentino cheese. Food Research International 40 (10) 1220-1226.

Zalazar C, Meinardi C. Hynes E. 1999. Quesos típicos Argentinos. Centro de Publicaciones Universidad Nacional del Litoral, Santa Fe. 